\title{
Editorial
}

\section{Participación, motivación y adherencia en Educación Física}

\author{
Javier A. Hall López, Paulina Y. Ochoa Martínez y Pedro Sáenz-López
}

\begin{abstract}
En el año 2008, Javier Hall, entonces profesor en la Universidad Autónoma de Sinaloa, solicitó al profesor Pedro Sáenz-López de la Universidad de Huelva una estancia de investigación financiada por la AUIP. Unos meses más tarde, Paulina Ochoa realizó otra estancia en la UHU. A partir de estas primeras actividades académicas de la que surgió un artículo (Hall, Ochoa, Sáenz-López, y Monreal, 2009), se han forjado unas relaciones cada vez más productivas basadas en la confianza y profesionalidad de docentes e investigadores de la UABC y la UHU. Estancias, proyectos y publicaciones en una red de relaciones imparable y creciente en la que docentes y alumnado de ambas instituciones y de otras están sacando provecho. Investigadores de ambas instituciones han colaborado en los proyectos de investigación que se estaban desarrollando en las respectivas universidades. El denominador común siempre ha sido la búsqueda de una Educación Física más eficiente y participativa que siente las bases de conseguir una población activa buscando la creación de hábitos saludables desde el sistema educativo. Este fue el gran objetivo del libro: "Hall, J. A., Ochoa, P. Y., y Sáenz-López, P. (2018) (Coords). Intensidad, salud, motivación y adherencia en Educación Física. Huelva: Servicio de Publicaciones de la Universidad de Huelva". Los artículos del presente número de e-Motion han salido de este libro con el objetivo de que tengan mayor difusión, al poderse publicar en abierto.
\end{abstract}

Los beneficios innegables de la práctica deportiva suponen, para numerosos expertos, el primer pilar de la medicina preventiva. Por tanto, conseguir jóvenes activos que adquieran una adherencia a la práctica de actividad físico-deportiva es un objetivo prioritario del sistema educativo. Es una necesidad social que los ministros y ministras de educación de todos los países se vayan convenciendo de aumentar el número de horas semanales de educación física. Mientras tanto, los docentes de esta asignatura podemos colaborar de dos formas: aumentar el tiempo de participación en las clases de educación física y conseguir una mayor adherencia a la práctica a través del aumento de la motivación intrínseca. Y sobre estas reflexiones se han escrito los artículos de este número que presentamos que desarrollan temáticas que van desde la descripción de la realidad del sedentarismo y obesidad infantil y juvenil como problemas crónicos en nuestros contextos, datos del nivel de participación en la clase de educación física evaluada mediante el Sistema para Observar el Tiempo de Instrucción de Actividad Física (SOFIT), hasta la explicación de las principales teorías sobre motivación y su aplicación práctica a la EF, así como a la necesidad de conseguir la adherencia de los jóvenes. De acuerdo con los estándares establecidos internacionalmente por el United States' National Association for Sport and Physical Education (NASPE), el índice de actividad física moderada a vigorosa (IAFMV) en una clase de EF debe ser de por lo menos el $50 \%$ del total. Para conseguirlo, se necesita un alumnado altamente motivado por lo que se han incorporado artículos que explican cómo conseguir aumentar la motivación intrínseca y la participación en las clases de educación física. 
El citado libro y los artículos aquí presentados son el fruto de un trabajo cooperativo de investigadores de la UABC y de la UHU. Agradecemos a las instituciones que han permitido estancias de investigación tanto en Mexicali como en Huelva: AUIP, Fundación Carolina de España y la Secretaria de Relaciones Exteriores de México, convocatorias de movilidad académica de la UABC y ayudas a estancias del Plan Propio de la UHU. Durante estos años, hemos compartido y apoyado mutuamente los proyectos en activo que existían en ambas Universidades. En este sentido, agradecemos al Ministerio de Educación de España la financiación del proyecto de investigación "Factores motivacionales relacionados con la práctica de actividad física y el bienestar psicológico" (Ref. DEP2011-28288). Al proyecto de Turismo Activo en Guadiana que financió el proyecto "estudio transfronterizo sobre Actividad Física, obesidad, alimentación e imagen corporal" a través de fondos POCTEP y FEDER. De la misma forma, este proyecto ha formado parte de una investigación denominada correlación de la intensidad, gasto energético y el esfuerzo físico del alumno y las proporciones del contexto y comportamiento del profesor al impartir clases de educación física California (Protocolo \#UABC-EXB-225) financiado por la Dirección de Superación Académica DAS-Secretaria de Educación Pública SEP en México.

Agradecemos la participación de los investigadores de México, España y Portugal a los que agrademos su calidad académica y humana en busca de una educación física moderna, innovadora, eficiente y saludable.

Dr. Javier A. Hall López ${ }^{1}$, Dra. Paulina Y. Ochoa Martínez y Dr. Pedro Sáenz-López²

${ }^{1}$ Universidad Autónoma de Baja California

${ }^{2}$ Universidad de Huelva

\section{REFERENCIAS BIBLIOGRÁFICAS}

Hall, J. A., Ochoa, P. Y., Sáenz-López, P., y Monreal, L. R. (2009). Estudio comparativo del nivel de actividad física, estado nutricio y obesidad abdominal en profesores de educación física de la Universidad Autónoma de Sinaloa y la Universidad de Huelva. Retos, 15, 5-8.

Hall, J. A., Ochoa, P. Y., y Sáenz-López, P. (2018) (Coords). Intensidad, salud, motivación y adherencia en Educación Física. Huelva: Servicio de Publicaciones de la Universidad de Huelva. 